\title{
Research on the Status and Development Trends of Embedded Software Technology
}

\author{
Changlin $\mathrm{He}^{1, \mathrm{a}}$, Yufen $\mathrm{Li}^{2, \mathrm{~b}}$ \\ ${ }^{1}$ Information Technology Service Center Hexi University, No.846 Beihuan Road, Zhangye, Gansu, \\ China. \\ ${ }^{2}$ ICC of Zhangye Power Supply Company, Zhangye, Gansu, China. \\ ahecl@hxu.edu.cn, ${ }^{b}$ liyufen626@163.com
}

Keywords: Embedded Software Technology, Status, Development Trends

\begin{abstract}
Currently, embedded technology has become a key technology of information industry, embedded technology has become an important part of computer technology development, but also been widely applied to aerospace, industrial production, military and national defense, health and our daily life, on the current state of development of embedded technology, software code having a smaller, high degree of automation, faster response, and other notable features, this paper embedded software technology related introduction to study the status of development of embedded technology and its future trends, in order to promote the further development of embedded technology, promoting the leaping development of China's information industry.
\end{abstract}

\section{Introduction}

With the rapid development of science and technology, we are gradually entering into the information-based societies, our lives and learning a lot of convenience. In our daily lives, computer network as a general thing has penetrated into every aspect of our lives, with the support of science and technology, the simple computer machine already difficult to meet our needs, followed by people of all pursuit of new types of embedded software and systems, in terms of our lives embedded software and systems, there is a TV home network, intelligent home appliances, GPS navigation systems, smart phones, MP3 and other digital intelligent miniaturized products, with respect to industrial production, the embedded software and systems mainly digitized production of machine tools, intelligent tools, computer as an important part of the information processing \& embedded technology, it is applied to a computer system or software which, namely embedded Some software technology is transformed into a curable component which is applied to the computer hardware system to achieve the perfect combination of computer hardware and computer software systems.

According to relevant data show that in 2008, the amount of software industry revenue reached 757 billion RMB, in which, embedded software revenue accounted for $14.8 \%$, accounting for nearly $2 / 3$ in the amount of software export revenue, in 2009, China Embed style software has been further developed, its market has reached nearly 250 billion RMB in 2011, the income scale of embedded software reached 470 billion RMB, creating a maximum amount of income over the years. On the current state of development of embedded technology, software code having a smaller, high degree of automation, faster response, and other notable features, so under the accelerating trend of social development, embedded technology in our lives, learning and the work will be more widely applied, but will also contribute to our economic development, greater strength.

\section{The Generation of Embedded Software}

Embedded software is based on embedded systems, embedded systems are often described as "application-centric", it is a form of a computer application, referring to burial in the host device microprocessor system. Such equipment is not generally computer users care about, also known as implantable computer. It mainly consists of four parts: embedded microprocessors, peripheral 
hardware devices, embedded operating system and application software system.

Starting from the above definition, embedded software features can be summarized in two words: "small", "special", "E", "Jane." The so-called "small" refers to special equipment based on common characteristics and needs, generally do not write too much; "special" means that it is generally based on the functional design of a system of separate write (or modify the existing software) a species highly personalized software product; "special" is not only from the perspective of a dedicated system for, and for system functions, system security and other aspects of the optimal configuration and power considerations, embedded software even within the same brand, same range of products also need to constantly be modified according to the system hardware changes and changes in, and this modification is clearly different from common software "upgrade"; As for the "Jane" is said embedded software is generally not required to design and function on realized too complicated, both for simple cost control purposes, but also for system security considerations.

Embedded software, the operating system is extremely important, it is the same with the PC, especially for embedded systems generally all software (operating system and application software) and the overall design of a one-time installation, so the embedded operating system position compared to PC actually more important and prominent.

Generally it believed that the rise of embedded software began 1970s, so far, 20 years of development history. But as the IT industry, economic forecasting and statistics in a separate category, it is emerged in recent years, and is limited to the United States and a few other countries. According to foreign research institutions in 1999, the embedded operating system of global output \$ 362 million, compared with \$ 305 million in 1998, an increase of 18.7\%. And the other research institutions is expected from 1997 to 2001, the embedded systems market will be 240\% of the average annual growth rate, market size in 2001 will reach \$ 31.7 billion. Currently, the embedded operating system, more varieties, statistics show a year ago, only for information appliances will have 40 embedded operating system species. The rapid expansion of the market over the past year, attracting more and more manufacturers enter the product categories is estimated that at least doubled. In many embedded operating system product to 3Com's Palm OS and Microsoft's Windows CE the most famous, they are no doubt the market leader in the competition, respectively, occupy $70 \%$ and $20 \%$ share of the handheld market.

\section{The Concept and Characteristics of Embedded Software}

The so-called embedded software is through some technical development of the operating system or other software embedded in hardware which, applied to the industrial production process is embedded in the software, the first to conduct chip design and development, re-design of embedded system software, and then proceeds to manufacture of embedded electronic devices among. Wherein the embedded system is a computer system with a stand-alone function, including the main memory, microprocessors, microcontrollers, etc., it is applied as a basis, emphasizing coordination between hardware and software, together to complete the program task.

The main features of embedded software are: practicality, usability refers to its functional complex needs of users, embedded software which is embedded into the computer system, but also for the computer system services, which are closely linked with the hardware, embedded type software development is mainly based on user demand, market demand-oriented, development-oriented industrial development orientation. Agility, flexibility mainly refers to the embedded software can be developed at any time, there is no fixed time limit, embedded software is relatively small, can easily be embedded in a computer system, and after embedding not affect the functionality of the computer system, with the continuous updating and development of embedded software, can also be constantly upgraded to meet the needs of users continue.

\section{The Situation Analysis of Embedded Software Technology}

From the current development process of embedded software technology point of view, there are many challenges in the current, which requires this technology is capable of binding a wide range of 
development needs to innovate to change. The current rate of scientific and technological development of China's relatively rapid in the embedded software that has been widely used in people's working lives, and learning and so provides a convenient [2]. Network technology under its own embedded software functions perfectly well in the role of the operating system, to provide technical support for small electronic devices, and low-cost HMI exchange miniaturization goals. In the application-level embedded software technology mainly in software applications and systems development level has an important manifestation.

From the applications embedded software point of view, in terms of communication engineering and consumer electronics and industrial production have a wide application. Communications engineering major smart phones and global positioning systems; consumer electronics is mainly digital television and digital cameras, home gateways and other aspects; in industrial production is mainly CNC machine tools and manufacturing plants and other aspects. In addition to financial transactions as well as health care and transportation also has a relatively wide range of applications.

\section{The Challenges Facing the Embedded Software Technology}

In information appliances as the representative of the Internet era of embedded products, not only to show a bright future for the embedded market, injected new life; the same time, embedded systems technology, especially software technology presented new challenges. This includes: support the growing functional density, flexible network connectivity, lightweight mobile applications and multimedia information processing. In addition, of course, we need to deal with more intense market competition.

The network has become an inevitable trend. For external networking requirements, equipment must be embedded with a communication interface, the corresponding need TCP / IP protocol stack software support; a new generation of embedded devices need to have IEEE1394, USB, CAN, Bluetooth or IrDA communication interfaces, as well as the need to provide appropriate communications networking protocol software and physical layer driver software.

Support small size, small electronic devices, micro-power, and low cost. To meet this feature, the appropriate requirements of embedded product designers reduce processor performance, memory capacity and limit reuse interface chip. This design requires a corresponding increase in the embedded software. For example, select the best programming model and continuous improvement algorithm, using Java. Therefore, it is necessary to have experienced software people, need more advanced embedded software development technologies such as Java, Web.

Provides sophisticated multimedia user interface. The reason why millions of embedded devices for the users willing to accept, one important factor is the affinity between them and the users, natural man-machine interface, such as drivers manipulate highly automated car mainly through diet steering wheel, pedals and a joystick. People interact with the information requirements of the terminal screen with GUI-centric multimedia interface. Handwriting input, voice dial, email and color graphics, the image has achieved initial results. At present, some advanced PDA on the display screen to write Chinese characters has been achieved, a short voice message published, but off the palm language simultaneous translation there is a great distance.

\section{Thinking of Development Trend of Embedded Software Technology}

Wireless Network. Widely used in embedded software technology is also explained in the role play more significant level, to be able to respond effectively to the international market competition, we need to upgrade the embedded software technology. Wherein in the development of wireless network operating system has gradually been applied in the course of future development, the mobile communication network can be provided on the rich multimedia data services, and support functionality and more mobile terminal devices more prominent. Capable third generation mobile communication system effectively play the advantages in research and development on the operating system of the wireless network to increase the intensity. In the continuous development of 
embedded devices, and increasing demand for network development, the application of wireless network technology will become an important development direction of embedded software, the current is relatively stable wireless EPOC operating system, which is mainly carried out by the preparation of $\mathrm{C}++$ then apply a comprehensive approach to object design low-end smart phones to fully functional personal communication device requirements can be effectively met.

AI. Since the development and application of embedded software technology is mainly for people to meet actual demand exists, so this needs to be upgraded in a timely manner in accordance with people's needs change, the service will fully reflect this feature. In artificial intelligence technology will be able to lower the actual demand for effectively satisfied, and embedded software technology has been effectively combined, the human-computer interaction to achieve this goal and to the greatest range of embedded software technology to be applied. Combined with the application in the health field of artificial intelligence technology and embedded software technology is relatively prominent, in particular the application process you will be able to effectively reduce patient harm. Application of artificial intelligence technology in the future will become an inevitable trend of development, for example in the life of intelligent automatic temperature control instruments and other equipment are presented artificial intelligence technology.

Network Interconnection. To be capable of embedded software technology distributed processing architecture of the Internet needs to be met, to be equipped with standard multi-network communication interface, a new generation of embedded devices are also required to provide the appropriate communications protocol software and physical layer network driver software. From the case of a conventional single-chip technology, the current actual demand has been unable to effectively meet, and in the development of a new generation of embedded software level on the good solution to this problem. Embedded processor on the embedded network interface can efficiently support TCP / IP protocol, but also to support the communication interfaces such as USB, embedded in the new technologies of communication network protocols and physical drivers are able to have easy access to the Internet, but also can effectively meet the needs of many Internet mobile user equipment. With the further development of the network technology, the optimization of embedded software technology will make aspects of people's work and life more convenient.

\section{Conclusions}

In summary, with the current development of China's science and technology, some of the more advanced technology in real life has been widely used, including in the development of embedded software technology, which is the most common PC computer time application form. In the era of rapid development of information technology, a simple computer machine on people's life and work demands are not met, so the use of embedded software and systems technology will be able to meet the actual application requirements to a large extent.

\section{Acknowledgment}

This work is supported by Teaching research project of Hexi University at Zhangye (No. HXXYJY-2014-017).

\section{References}

[1] Huifang Zhou: Computer Application, Vol. 6 (2004) No 53, p.25-26

[2] Hongli Zhang: Information Technology, Vol. 12 (2005) No 27, p.74-76

[3] Qin Guo: Computer and Network, Vol. 1 (2006) No 33, p.11-14

[4] Jieming Liu: Guangxi Normal University, Vol. 3 (2007) No33, p.121-124

[5] Qiliang Hu: Guangxi Sciences Academy, Vol.21 (2010) No18, p.60-64 